\title{
Comportamento da coluna total de dióxido de enxofre na camada limite da região da cidade de Santiago no Chile utilizando o sensor OMI
}

\author{
The total column behavior of sulfur dioxide in the boundary layer in the region of the city of \\ Santiago in Chile using OMI sensor
}

\author{
Mateus Dias Nunes ${ }^{1}$, Éricka Voss Chagas Mariano² e Glauber Lopes Mariano ${ }^{3}$ \\ ${ }^{1}$ Mestrando, Universidade Federal de Pelotas, Pelotas, Brasil \\ nunes.mateusdias@gmail.com \\ ${ }^{2}$ Doutor, Faculdade de Meteorologia, Universidade Federal de Pelotas, Pelotas, RS, Brasil \\ erickavoss@gmail.com \\ ${ }^{3}$ Doutor, Faculdade de Meteorologia, Universidade Federal de Alagoas, Maceió, AL, Brasil \\ glaubermariano@gmail.com
}

\begin{abstract}
Resumo
A poluição atmosférica é um dos principais problemas ambientais do mundo atual. O gás dióxido de enxofre $\left(\mathrm{SO}_{2}\right)$ um poluente estudado tanto em áreas remotas como em áreas urbanas, podendo ser emitido na atmosfera através de fontes naturais e também por atividades antropogênicas, sendo seu estudo de grande relevância para o monitoramento de problemas causados à saúde da população, agricultura e problemas econômicos principalmente aos centros populacionais de grande e médio porte. O objetivo deste estudo foi determinar a variabilidade da coluna total de dióxido de enxofre para a região da cidade de Santiago no Chile utilizando dados do sensor OMI (Ozone Monitoring Instrument) que opera dentro da faixa do UV/VIS para o período de 2005 a 2012. Utilizando os valores máximos diário, da coluna total de SO2, determinou-se da variabilidade anual e sazonal da coluna total de dióxido de enxofre para a região de Santiago no Chile. A Média Móvel para 30 dias (MM30) não apresentou uma sazonalidade particular para o período de 2005 a 2012. Foi possível observar valores crescentes da linha de tendência na dispersão dos dados da região de Santiago.
\end{abstract}

Palavras-chave: Dióxido de enxofre, Sensor OMI, Coluna total.

\begin{abstract}
Air pollution is a major environmental problem of today's world. The sulfur dioxide gas $\left(\mathrm{SO}_{2}\right)$, a pollutant studied both in remote areas as in urban areas, and can be emitted into the atmosphere through natural sources and also by human activities, and its study of great relevance for monitoring problems caused to health of the population, agriculture and economic problems mainly to the population centers of large and medium size. The objective of this study was to determine the variability of the total column of sulfur dioxide into the region of the city of Santiago in Chile using data from the sensor OMI (Ozone Monitoring Instrument) which operates within the range of UV / VIS for the period 2005-2012. Using the daily maximum values of total $\mathrm{SO}_{2}$ column, it was determined the annual and seasonal variability of the total column of sulfur dioxide into the region of Santiago in Chile. The Moving Average for 30 days (MM30) was not a particular seasonality for the period 2005-2012. It was observed increasing values of trend line in the dispersion of the data of the Santiago region.
\end{abstract}

Keywords: Sulfur dioxide, Sensor OMI, Column total. 


\section{Introdução}

A poluição atmosférica é um dos principais problemas ambientais do mundo atual. $\mathrm{O}$ gás dióxido de enxofre $\left(\mathrm{SO}_{2}\right)$ é usualmente um dos poluentes mais estudados, pois o mesmo é emitido tanto em áreas remotas como em áreas urbanas. Sua emissão para a atmosfera ocorre através de fontes naturais como, por exemplo, a erupção vulcânica e também causada por atividades antropogênicas como, por exemplo, a queima de combustíveis fósseis (MARIANO et al., 2014).

A maioria das espécies de gases emitida para a troposfera possui um tempo de vida curto e são destruídas dentro da própria troposfera, como por exemplo, $\mathrm{O} \mathrm{SO}_{2}$ que tem um tempo de vida de um a quatro dias (SCHROEDER e LANE, 1998). Entretanto, mesmo com seu tempo de vida curto, partículas relativamente leves (como as oriundo de dióxido de enxofre) podem circular e dispersar tanto horizontalmente quanto verticalmente conforme a orientação dos ventos além de interagir com outros poluentes (fase vapor) e aerossóis (MARTINS e ANDRADE, 2002).

Uma vez emitido na atmosfera, o $\mathrm{SO}_{2}$ pode reagir com vários oxidantes presentes na atmosfera tornando-se formador de sulfato particulado, como na forma de gotas de $\mathrm{H}_{2} \mathrm{SO}_{4}$ (ácido sulfúrico) ou até mesmo na forma de partículas neutralizadas tais como sulfato de amônio $\left(\left(\mathrm{NH}_{4}\right)_{2} \mathrm{SO}_{4}\right)$ (LEE et al., 2011). Em certas condições, $\mathrm{O}_{2} \mathrm{SO}_{2}$ pode transformar-se em $\mathrm{SO}_{3}$ (trióxido de enxofre) e, com a umidade atmosférica, transformar-se em $\mathrm{H}_{2} \mathrm{SO}_{4}$, sendo assim um dos componentes precursores para a formação da chuva ácida. Também tem grande significância no processo físico da formação das nuvens, aumentando a refletividade das mesmas (BEKKI, 1995). Quando chega à região estratosférica, principalmente catapultados por emissões vulcânicas, recombina-se com a água, assim formando aerossóis de sulfato que podem agir como núcleos de condensação de nuvens que por sua vez têm seu albedo (coeficiente de reflexão) alterado fazendo com que a incidência de radiação de onda curta diminua na superfície da Terra resultando no seu resfriamento (LIOU, 2002).
Wang et al. (2006), definem que medições de emissões, dispersão e comportamento do total de $\mathrm{SO}_{2}$ na coluna atmosférica tanto podem ser feitas através de sensores a bordo de satélites (ex.: OMI - Ozone Monitoring Instrument, TOMS - Total Ozone Mapping Spectrometer, GOME - Global Ozone Monitoring Experiment) quanto por instrumentos de superfície (ex.: Espectrofotômetro de Brewer). Operando desde agosto de 2004 a bordo do satélite Aura da NASA (National Aeronautics and Space Administration), o sensor OMI, projetado para monitorar ozônio e outras espécies de gases, realiza entre outras funções, medições diárias globais da coluna total de $\mathrm{SO}_{2}$ em diversos níveis (KROTKOV et al., 2006). Quantitativamente, o $\mathrm{SO}_{2}$ antropogênico é mais difícil de medir do espaço, já que a absorção de ozônio e espalhamento Rayleigh reduz a sensibilidade aos poluentes na baixa troposfera (YANG et al., 2007). Devido a essas características mencionadas, o sensor OMI torna-se mais sensível ao $\mathrm{SO}_{2}$ acima das nuvens e da neve (gelo) e menos sensível ao $\mathrm{SO}_{2}$ abaixo das nuvens (KROTKOV et al., 2008). Devido à sensibilidade reduzida do $\mathrm{OMI}$ para $\mathrm{SO}_{2}$ próximo a superfície, este produto deve ser usado somente sob condições de visualização ideal (sem a presença de nuvens, nevoeiros e etc).

Algumas das principais regiões metropolitanas do sul da América do Sul apresentam forte poder econômico devido ao crescimento populacional, ao desenvolvimento industrial nas cidades e regiões metropolitanas de grande e médio porte (IBGE,2010).

Santiago, a capital chilena, como qualquer outra grande cidade, produz poluição, o que vem gerando a acumulação de uma capa de Smog fotoquímico, devido ao grande volume populacional, aumentando as emissões através de atividades antropogênicas. Esta característica se agrava durante o inverno, devido aos fenômenos climáticos típicos do período (inversões térmicas, presença de centros de alta pressão, etc.). Tudo isso, juntamente com valores de temperatura mais amena próprios dessa época do ano, produzem o aumento das infecções respiratórias (NUNES et al., 2013).

O objetivo geral deste trabalho é determinar e diagnosticar o comportamento e a variabilidade da coluna total de dióxido de enxofre na região 
da cidade de Santiago no Chile para o período de 2005 a 2012 dentro da Camada Limite Planetária (CLP). Através da variabilidade da concentração da coluna de $\mathrm{SO}_{2}$ na atmosfera, poderá se conhecer as características da região do estudo, com altos valores de concentração total de $\mathrm{SO}_{2}$.

\section{Metodologia}

\subsection{Dados do sensor OMI}

Neste trabalho foram utilizados os dados diários da concentração total de dióxido de enxofre na coluna atmosférica correspondente a CLP na região metropolitana da cidade de Santiago no Chile $\left(33^{\circ} 45^{\prime} \mathrm{S}, 70^{\circ} 65 \mathrm{~W}\right)$ durante o período de janeiro 2005 a dezembro 2012, obtidos através do sensor OMI a bordo do satélite Aura. Para delimitarmos as áreas de estudo nas diferentes regiões e devido a possíveis falhas varredura dos dados obtidos, utilizou-se um incremento de $1,0^{\circ}$ na latitude e $1,0^{\circ}$ de longitude de cada ponto observado.

Os dados utilizados estão disponíveis no site da NASA em (http://so2.gsfc.nasa.gov). O sensor OMI opera dentro da faixa do UV/VIS entre 306380nm (nanômetros), realizando varreduras diárias em órbita da Terra captando informações de dados com uma resolução espacial de $0,125^{\circ} \mathrm{x}$ $0,125^{\circ}$ no nível L2G, baseados na extinção de Radiação de Onda Longa (ROL) ascendente que é proveniente da Radiação Infravermelha (IR) refletida pelo sistema Terra-atmosfera (KROTKOV et al., 2008).

Utilizou-se o nível L2G, nível que representa as emissões antropogênicas provenientes de atividades industriais e emissões veiculares referente à recuperação do $\mathrm{SO}_{2}$, por apresentar melhor qualidade na base de dados. Este nível é gerado a partir do nível L2 onde é desconsiderada espessura ótica da camada, cobertura de nuvens, albedo da superfície entre outros fatores. Dessa forma, os dados passaram por teste de qualidade caso contrário seriam eliminados (cerca de 30\% dos dados obtidos são eliminados) (KROTKOV et al., 2006). Usa-se a altura da pluma de $\mathrm{SO}_{2}$ do centro de massa referente ao nível da camada limite planetária, corresponde à altitude em torno de $0,9 \mathrm{~km}$. Utilizou-se o nível de observação na camada limite planetária da coluna total de $\mathrm{SO}_{2}$, por esta ser a camada que sofre maior influência antropogênica.

Semelhante à Fioletov et al., (2011) que utilizaram os valores máximos diários de dióxido de enxofre, estabeleceu-se um limiar máximo para o total de dióxido de enxofre na coluna atmosférica de 5,0 UD (Unidade Dobson). Portanto, eliminou-se valores superiores a 5,0 UD e inferiores a zero, sendo os registros de valores negativos e acima de 5,0 UD considerados como possíveis falhas nas medições do sensor, sendo tratados como valores nulos.

Aplicando-se essa metodologia resultou-se na exclusão de 292 (duzentos e noventa e dois) casos para a região da cidade de Santiago no Chile durante o período analisado.

\subsection{Classificação dos extremos a partir do percentil 99\% (P99)}

Os casos extremos de máxima concentração da coluna total de $\mathrm{SO}_{2}$ foram identificados através do percentil 99\% (P99), que indicam onde se concentram os $1 \%$ dos casos extremos encontrados, tanto anualmente, sazonalmente quanto mensalmente durante o período de dados. A análise anual ocorreu a partir da quantificação dos casos onde ocorreram os valores extremos (valores acima do P99) para a região escolhida.

Para compreender e analisar a distribuição mensal dos valores diários do total de dióxido de enxofre na coluna atmosférica, mostrando onde estão alocados os dados através de mediana e quartil, utilizaram-se gráficos boxplot. O comportamento dos dados dentro do período de estudo também foi analisado através de média móvel com período igual 30 dias (MM30) (WILKS, 2006).

\section{Resultados e Discussões}

\subsection{Análises da dispersão dos dados para o período de 2005 a 2012}

Através dos dados analisados para o período de 2005 a 2012, foi constatado um valor de 4,78 UD do P99 para a região da cidade de Santiago. A 
configuração da reta de regressão linear mostra onde se concentra a tendência dos dados para a região da cidade de Santiago, com valores entre 1,7 UD e 2,2 UD. A região de Santiago apresenta os valores máximos da coluna total de dióxido de enxofre estando mais concentrados abaixo da reta de regressão linear do que nos valores acima da reta de regressão. Não há uma configuração exata da MM30 no período de 2005 a 2012 para as transições entre os anos ou entre as estações. Já no último ano da série é possível observar um forte decaimento na quantidade de dados de valores máximos da coluna total de dióxido de enxofre devido aos valores falhos (negativos ou valores ausentes) e valores acima do limiar empregado de 5 UD de acordo com a Figura 1.

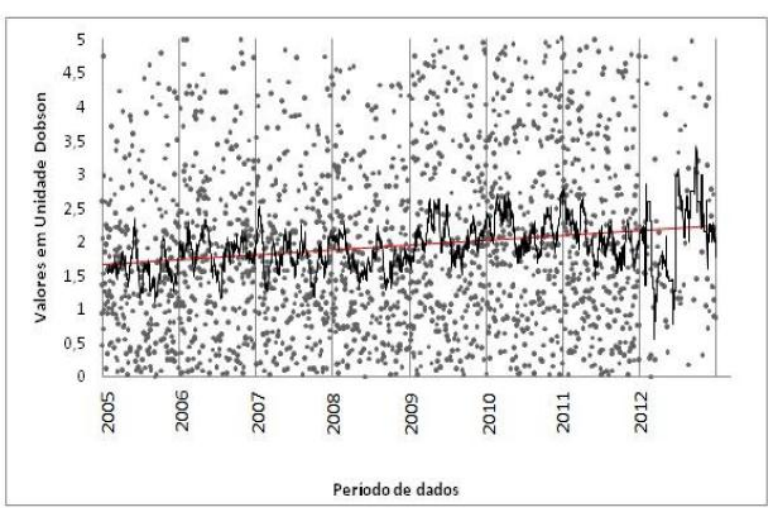

Figura 1 - Dispersão, linha tendência e média móvel dos dados de valores máximos diários da coluna total de $\mathrm{SO}_{2}$ na região Santiago no período de 2005 a 2012

Na investigação do histograma representativo à região de Santiago (Figura 2) é possível constatar que as maiores frequências dos dados se concentram nos quatro primeiros intervalos de classes, sendo mais de $60 \%$ dos dados onde os valores se concentram até 2,0 UD, o que faz com que a série de dados não siga uma assimetria Gaussiana tendo a sua assimetria positiva concentrando os dados mais à esquerda da mediana, onde o terceiro intervalo apresentou uma frequência com um valor de 310 casos e o quarto intervalo de classe apresentou uma frequência com um valor de 320 casos, que somados são mais de $35 \%$ do total de dados. Percebe-se que a partir do quinto intervalo de classe há um decréscimo da freqüência absoluta em direção aos últimos intervalos de classe. A menor freqüência se dá no último intervalo de classes, que aborda a faixa de 5,0 UD, tendo sua freqüência de 67 (sessenta e sete) registros, cerca de $3 \%$ dos casos, o que comprova que a maior quantidade de casos (97\%) ocorre abaixo do P99 (4,78 UD) para a cidade de Santiago.

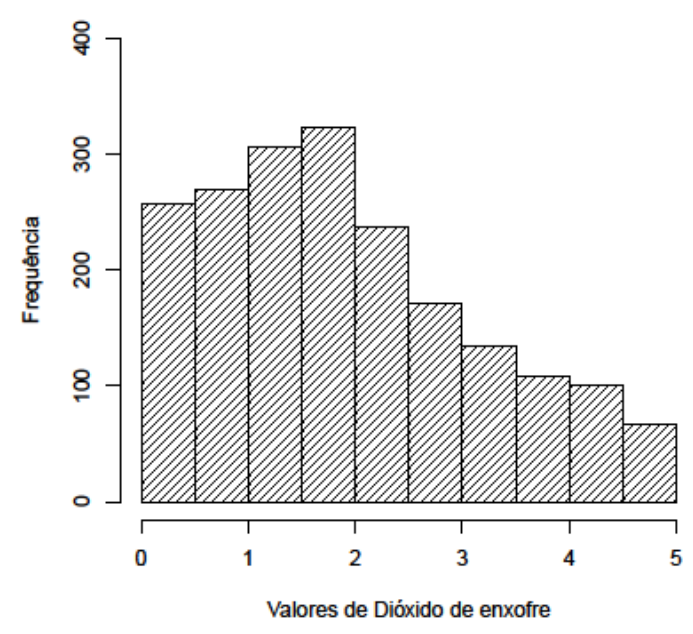

Figura 2 - Histograma das distribuições de freqüências dos dados para o período de 2005 a 2012 para a região de Santiago no Chile

\subsection{Análise interquartílica mensal dos máximos valores diários de dióxido de enxofre}

De acordo a Figura 3, a análise do período de 2005 a 2012 indica que a região apresentou um padrão sem grandes alterações entre os meses dentro do período analisado, tendo mediana entre 1,1 UD de valor mínimo e 1,8 UD de valor máximo mostrando que não existe nenhuma variação significativa entre os meses. $\mathrm{O}$ valor da mediana entre os meses de agosto e dezembro apresentam uma leve variação em relação ao período de janeiro a abril, onde a mediana não apresenta um padrão tão linear para os valores máximos da concentração da coluna total de $\mathrm{SO}_{2}$ onde podemos encontrar valores que chegam à maior distância para essa região que é de 0,7 UD. Tanto os valores dos quartis inferiores e superiores não apresentam nenhuma variação significativa entre os meses, porém observa-se um comportamento quase constante desses valores. Dois casos com os valores discrepantes à se (outliers) à série de dados foram encontrados nos meses de novembro. 


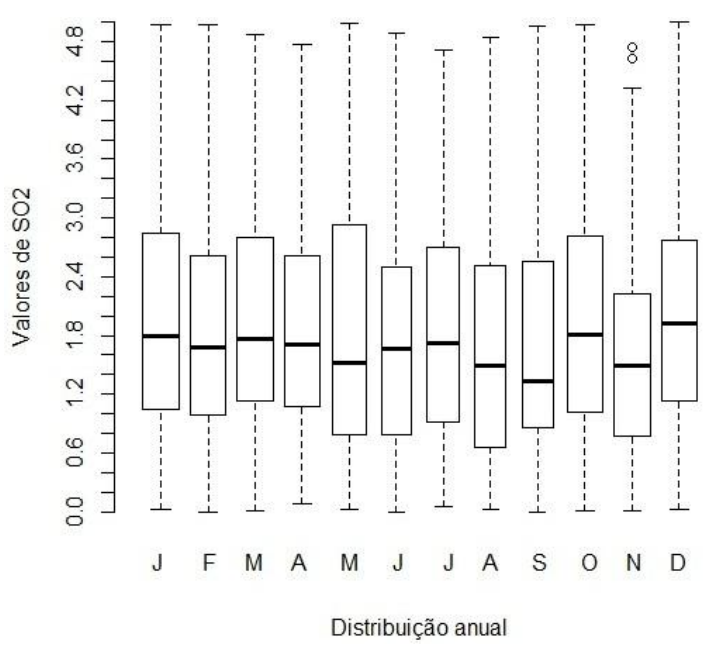

Figura 3 - Distribuição mensal dos máximos valores diários da coluna total de dióxido de enxofre para o período de 2005 a 2012

\section{Conclusões}

Após análise dos resultados dos valores da coluna total de enxofre referente à camada limite planetária da região de Santiago no Chile percebe-se que os valores da linha de tendência mantêm-se entre 1,7 UD e 2,2 UD e a MM30 não apresentando uma sazonalidade particular para o período de 2005 a 2012. Foi possível observar valores crescentes da linha de tendência na dispersão dos dados da região de Santiago indicando tendência de aumento dos valores de máxima concentração de dióxido de enxofre na região.

Observou-se um padrão de frequência dos eventos de máxima concentração nos quatro primeiros intervalos de classe (cerca de 60\%) até 2,0 UD, o que concorda com a faixa onde se encontra a reta de regressão linear.

$\mathrm{Na}$ análise interquartílica, observou-se que os valores da mediana têm um padrão entre 1,2 UD e 1,8 UD. O mesmo ocorre entre os quartis inferiores e superiores para todos os meses do ano.

A importância de estudos sobre emissões de gases como o dióxido de enxofre é de extrema relevância quando se trata da análise de chuva ácida que pode causar danos importantes para a população. Os altos valores na coluna total de $\mathrm{SO}_{2}$ na atmosfera podem agravar e proporcionar problemas respiratórios a população sendo seu monitoramento de extrema importância.

\section{Agradecimentos}

Os Autores agradecem à Coordenação de Aperfeiçoamento de Pessoal de Nível Superior (CAPES) pelo apoio financeiro.

\section{Referências}

BEKKI, S. (1995). Oxidation of volcanic SO2: a sink for stratospheric $\mathrm{OH}$ and $\mathrm{H} 2 \mathrm{O}$. Geophysical Research Letters, 22: 913 - 916.

FIOLETOV, V.E.; MCLINDEN, C.A; KROTKOV, N.A.; MORAN, M.D; YANG, K. (2011). Estimation of $\mathrm{SO} 2$ emissions using OMI retrievals. Geophysical Research Letters, 32: 1 - 4.

IBGE. (2010). Instituto Brasileiro de Geografia e Estatística http://www.ibge.gov.br/ (acessado em 20 de janeiro de 2015).

KROTKOV, N.A.; CARN, S.A.; KRUEGER, A.J.; BHARTIA, P.K.; YANG, K. (2006). Band residual difference algorithm for retrieval of $\mathrm{SO} 2$ from the Aura Ozone Monitoring Instrument (OMI). IEEE Transactions Geoscience and Remote Sensing, 44: $1259-1266$.

KROTKOV, N.A.; MCCLURE, B.; DICKERSON, R.R.; CARN, S.S.; LI, C.; BHARTIA, P.K.; YANG, K.; KRUEGER, A.J.; LI, Z.; LEVELT, P.F.; CHEN, H.; WANG, P.; LU, D. (2008). Validation of SO2 retrievals from the Ozone Monitoring Instrument (OMI) over NE China. Journal of Geophysical Research, 113: D16S40, doi:10.1029/2007JD008818.

LEE, C.; MARTIN, R.V.; VAN DONKELAAR, A.; LEE, H.; DICKERSON, R.R.; HAINS, J.C.; KROTKOV, N.; RICHTER, A.; VINNIKOV, K.; SCHWAB, J.J. (2011). SO2 emissions and lifetimes: Estimates from inverse modeling using in situ and global, space-based (SCIAMACHY and OMI) observations. Journal of Geophysical Research, 116: D06206, doi:10.1029/2010JD014758.

LIOU, K.N. (2002). An introduction to atmospheric radiation. Califórnia: Academic Press. 583p. 
MARIANO, E.V.C. (2012). Desenvolvimento de metodologia para validação e análise de dados de dióxido de enxofre (SO2) atmosférico sobre o Continente Sul-americano e região metropolitana subantártica. Programa de Pós-graduação em Geofísica Espacial - Instituto Nacional de Pesquisas Espaciais, São José dos Campos. Tese de Doutorado, 89p.

MARIANO, E.V.C; NUNES, M.D; MARIANO, G.L. (2014). Dióxido de enxofre atmosférico fontes, medições, monitoramento e impactos na América do Sul. Em: MARIANO, G.L (ed.). Meteorologia em Tópicos. Clube dos Autores, p. 47-95.

MARTINS, C.R.; ANDRADE, J.B. (2002). Química atmosférica do enxofre (IV): emissões, reações em fase aquosa e impacto ambiental. Química Nova, 25: 259 - 272.

NUNES, M.D; MARIANO, G.L; MARIANO, E.V.C. (2013) Variabilidade da coluna total de dióxido de enxofre sobre o sul da América sul utilizando dados do sensor OMI Em: VIII Workshop Brasileiro de Micrometeorologia.

SCHROEDER, W.H.; LANE, D.A. (1998). The fate of Toxic Airborne Pollutants. Enviromental Science Technology, 2: 240 - 246.

YANG, K.; KROTKOV, N.A.; KRUEGER, A.J.; CARN, S. A.; BHARTIA, P.K.; LEVELT, P.F. (2007). Retrieval of large volcanic SO2 columns from the Aura Ozone Monitoring Instrument: Comparison and Limitations. Geophyscal Research, 96: 711 - 20.

WANG, P.; RICHTER, A.; BRUNS, M.; BURROWS, J. P.; SCHEELE, R.; JUNKERMANN, W.; HEUE, K. P; WAGNER, T.; PLATT, U.; PUNDT, I. (2006). 86 Airborne multi-axis DOAS measurements of tropospheric $\mathrm{SO} 2$ plumes in the Povalley, Italy. Atmospheric Chemistry and Physics, 6: 329 - 338.

WILKS, D.S. (2006). Statistical Methods in the atmospheric science. California, Academic Press. $676 \mathrm{p}$. 